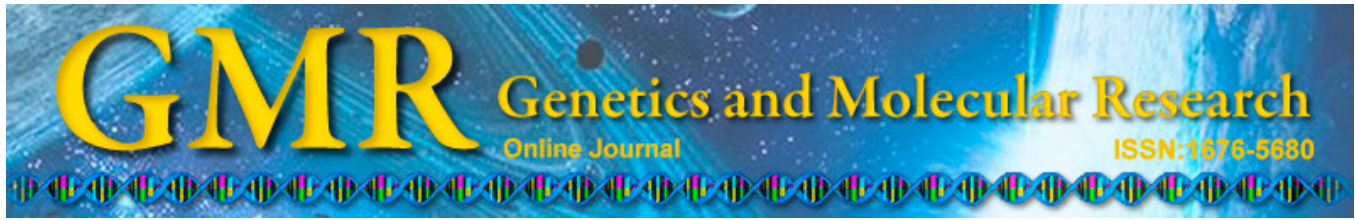

\title{
Behavior of calf Sertoli cells and fibroblast cells transfected with the human $H N P-1$ gene
}

\author{
N. Yu, A. Khalid, Y. Shi, X. Liu, X. Lin, Y.L. Li and G.X. Zhang \\ College of Animal Science and Technology, \\ Northeast Agricultural University, Harbin, China \\ Corresponding author: G.X. Zhang \\ E-mail: gxzhang@neau.edu.cn
}

Genet. Mol. Res. 13 (4): 9656-9664 (2014)

Received January 7, 2014

Accepted May 20, 2014

Published November 14, 2014

DOI http://dx.doi.org/10.4238/2014.November.14.11

\begin{abstract}
Human neutrophil peptide-1 (HNP-1) is an important defense molecule in neutrophils and Sertoli cells and plays an important role in the blood-testis barrier. In this study, we investigated the behavior of Sertoli cells transfected with the $H N P-1$ gene and compared the ability of Sertoli cells and fibroblast cells to resist transfection. Total RNA was isolated from human blood. The DNA coding sequence of $H N P-1$ was amplified by reverse transcription-polymerase chain reaction (RT-PCR) and the eukaryotic expression vector pEGFP-N3-HNP-1 was identified by PCR, endonuclease digestion, and sequencing. Bovine Sertoli cells and fibroblast cells were transfected with pEGFP-N3-HNP-1 using Liposome reagent. The transfection efficiency and the behavior of the transfected cells were evaluated at 24,48 and $72 \mathrm{~h}$ as well as at other times after transfection. The plasmid pEGFP-N3-HNP-1 was successfully constructed. The cells achieved maximum transfection efficiency at $48 \mathrm{~h}$. Two weeks after transfection, the cells began to stop dividing. The ability of Sertoli cells to resist transfection was higher compared to fibroblast cells. The ability of the 2 cell types to resist transfection was higher with plasmid pEGFP-N3-HNP-1 than with the plasmid pEGFP-N3. The injury to Sertoli cells caused by transfection with the $H N P-1$ gene was less pronounced than in fibroblast cells,
\end{abstract}


which may be closely correlated with the physiological function of Sertoli cells.

Key words: Calf; Fibroblast cell; Human HNP-1; Sertoli cell; Transfection

\section{INTRODUCTION}

Mammalian defensins are small antimicrobial peptides of 29-34 amino acids and are rich in cysteine and arginine ( $\mathrm{Lu}$ and de Leeuw, 2013). Defensins were first identified by Lehrer et al. (1980), following separation and purification from rabbit alveolar macrophages. These peptides can effectively kill Gram-positive and Gram-negative bacteria, certain fungi, spirochetes, and enveloped viruses and play an important role in antiviral immunity (Wilde et al., 1989; Lu and de Leeuw, 2013). Human neutrophil peptide-1 (HNP-1) is an important defense molecule in neutrophils. In 1994, the HNP-1 cDNA was restructured into the pBabe/ neo plasmid vector and transfected into mouse macrophages that did not contain endogenous defensin for in vitro culture (Yan and Chen, 2001). Because Sertoli cells are non-immune cells, they are widely applicable in the field of transplantation; in addition, these cells induce inflammatory cell infiltration to prevent the attack of allosome cells (Yu and $\mathrm{Hu}, 2010$ ). Thus, we transfected the recombinant eukaryotic expression plasmid pEGFP-N3-HNP-1 into in vitrocultured bovine Sertoli cells and fibroblast cells using the liposome transfection method. We examined the ability of Sertoli cells to resist transfection compared to fibroblast cells, determined cellular behaviors, and provide background for the development of transgenic animals with a high anti-infection ability and no rejection reaction.

\section{MATERIAL AND METHODS}

\section{HNP-1 purification}

Total RNA was extracted from human blood using cold Trizol reagent (Invitrogen; Carlsbad, CA, USA). Total RNA expression was analyzed by reverse transcription-polymerase chain reaction (RT-PCR) using the high-capacity cDNA Reverse Transcription Kit (Applied Biosystems; Foster City, CA, USA). The glyceraldehyde 3-phosphate dehydrogenase gene was used as a control. Primers were designed based on the HNP-1 cDNA sequence (No. NM_004084.3) in the GenBank database using the Premier 5.0 and the oligo6 software. Restriction site for EcoRI and BamHI were included on the forward and reverse primers, respectively. Primers were synthesized by the BGI Tech Company (Beijing, China); primer sequences are shown in Table 1.

PCR was performed as follows: pre-denaturation for $5 \mathrm{~min}$ at $94^{\circ} \mathrm{C}$, denaturation for $30 \mathrm{~s}$ at $94^{\circ} \mathrm{C}$, annealing for $30 \mathrm{~s}$ at $58^{\circ} \mathrm{C}$, and extension for $40 \mathrm{~s}$ at $72^{\circ} \mathrm{C}$; a total of 30 cycles was used, followed by extension for $10 \mathrm{~min}$ at $72^{\circ} \mathrm{C}$. After purification, the PCR amplification product was cloned into the pMD18-T vector (Takara; China) incubated at $16^{\circ} \mathrm{C}$ overnight, then transformed into Top 10 competent cells (Takara; Shiga, Japan). Positive recombinant plasmids were screened by ampicillin and PCR, digested by endonuclease, and sequenced by the BGI Tech Company. 


Table 1. Information of the primers.
\begin{tabular}{llc}
\hline Primer & Sequence & Product (bp) \\
\hline GAPDH-upper & 5'-AgCCACATCgCTCAgACAC-3' & 472 \\
GAPDH-lower & 5'-gAggCATTgCTgATgATCTTg-3' & 472 \\
HNP-1-upper & 5'-ggAATTCTATgAggACCCTCCCATCCTTgCTgC-3' & 294 \\
HNP-1-lower & 5'-CgggATCCgCAgCAgAATgCCCAgAgTCTTC-3' & 294 \\
\hline
\end{tabular}

\section{Construction of eukaryotic expression vectors pEGFP-N3-HNP-1}

The eukaryotic expression vectors pEGFP-N3 and pMD18-T-HNP-1 were digested by $E c o \mathrm{RI}$ and $B a m \mathrm{HI}$ at $37^{\circ} \mathrm{C}$ for $3 \mathrm{~h}$. The $H N P-1$ and $p E G F P-N 3$ fragments were ligated into the vectors by T4 DNA ligase. These products were amplified, the plasmid was extracted using the Endo-free Plasmid Mini Kit II (Omega Bio-Tek; Norcross, VA, USA), and the sample was preserved at $-20^{\circ} \mathrm{C}$ until transfection.

\section{Cell culture and transfection of the plasmids pEGFP-N3-HNP-1 and pEGFP-N3}

The skin tissue of 2-3-month fetal bovine and newborn bovine testis were recovered and transported to the lab within $4 \mathrm{~h}$ from the slaughterhouse at Harbin Modern Biological Technology Co., Ltd. (Harbin, Japan). Primary cultures of Sertoli and fibroblast cells were conducted as reported previously (Zheng et al., 2012; Yu et al., 2013). The pEGFP-N3-HNP-1 plasmid was transfected into Sertoli and fibroblast cells using Lipofectamine 2000 (Life Technologies; Carlsbad, CA, USA); the pEGFP-N3 plasmid was used as a positive control. Transfection efficiency and expression were evaluated at 24,48 , and $72 \mathrm{~h}$ under a fluorescence microscope. Total RNA was extracted from the 2 cell types at $48 \mathrm{~h}$ after transfection using Trizol reagent. Following RT-PCR and PCR, the products were evaluated by $1.5 \%$ agarose gel electrophoresis. Next, $600 \mu \mathrm{g} / \mathrm{mL}$ G418 was added to screen for positive cells and the culture medium was changed every 3-4 days. The behaviors of the transfected cells were observed for more than 10 weeks. Cell culture and transfection experiments for the pEGFP-N3-HNP-1 and pEGFP-N3 plasmids were repeated 6 times.

\section{Statistical analysis}

The transfection efficiency was considered as the percentage of positive cells in 1000 cells. Cell survival time was the period after which $90 \%$ of positive cells were dead after 2 weeks of transfection. All results were counted under a fluorescent inverted microscope (magnification $400 \mathrm{X}, 20$ vision random) using the mean \pm standard deviation to express the experimental data using the CoStat software.

\section{RESULTS}

HNP-1 gene cloning, eukaryotic expression vector pEGFP-N3-HNP-1 construction, and identification

The 294-bp specific amplification band was obtained by RT-PCR and PCR (Figure 1), 
confirming that the product was the $H N P-1$ gene sequence. Thus, the $H N P-1$ gene was successfully cloned. A diagram for construction of the eukaryotic expression vector pEGFP-N3HNP-1 is shown in Figure 2. Two fragments were obtained $(4.7+0.3 \mathrm{~kb})$ after double-digestion of the plasmid with EcoRI and BamHI (Figure 3). The size and direction of the fragments were correct according to the sequencing results as conducted by the Beijing BGI Tech Company. Thus, the pEGFP-N3-HNP-1 plasmid was successfully constructed.

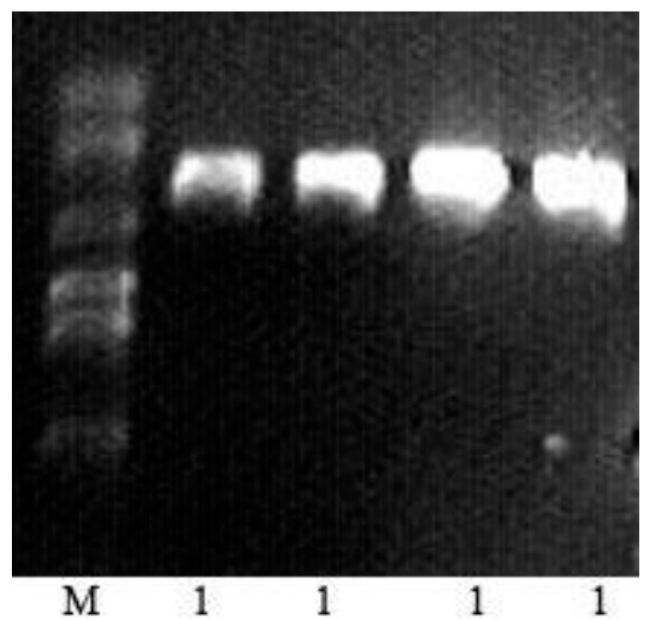

Figure 1. Cloning and identification of the HNP-1 gene. Lane $M=$ DL2000 marker; lane $1=H N P-1$.

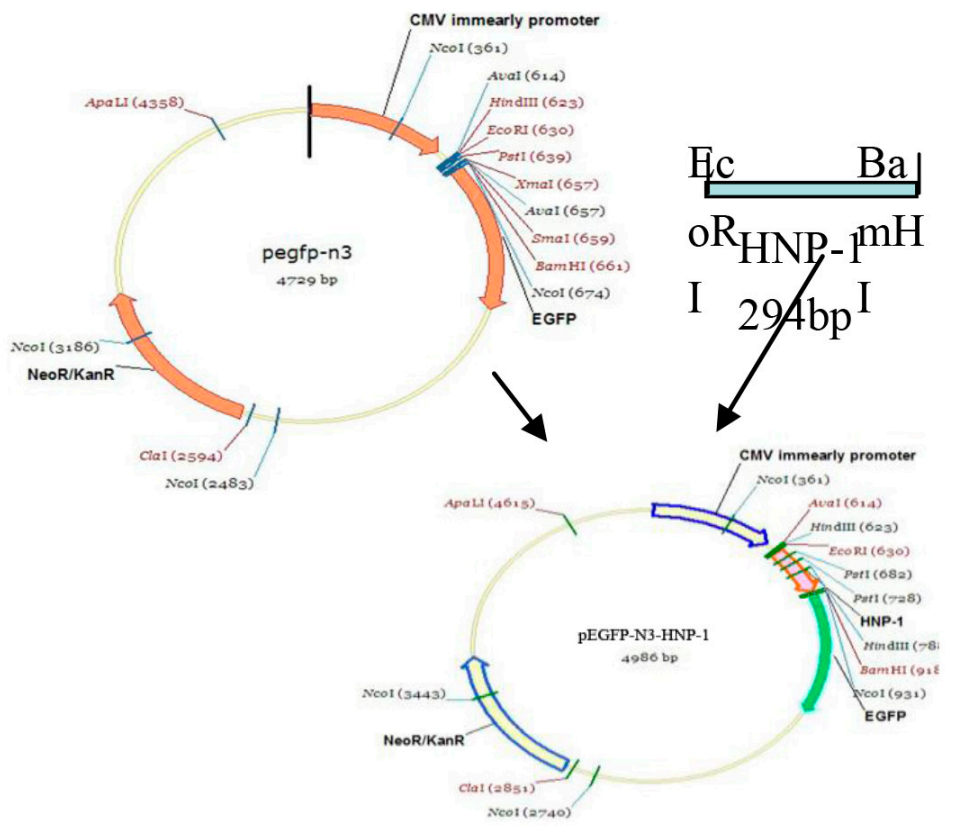

Figure 2. Map of plasmid pEGFP-N3-HNP-1 construction. 


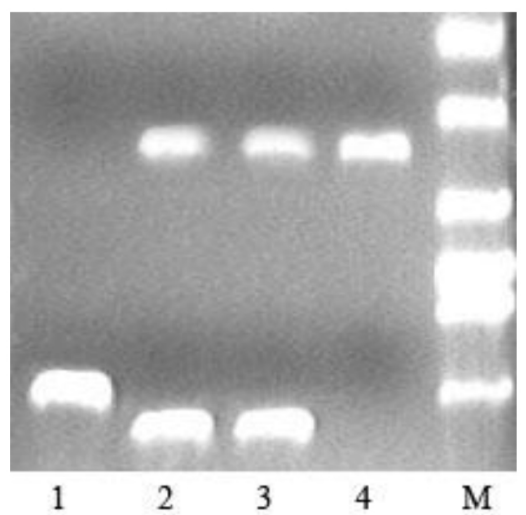

Figure 3. Double digestion of recombinant plasmid pEGFP-N3-HNP-1 by EcoRI and BamHI. Lane $1=$ plasmid of pEGFP-N3; lanes 2-3 = recombinant plasmid pEGFP-N3-HNP-1; lane $4=\mathrm{HNP}-1$; lane $M=$ DL2000 marker.

\section{Testing of transfected $H N P-1$ gene mRNA expression product}

At $48 \mathrm{~h}$ after transfecting the plasmids pEGFP-N3-HNP-1 and pEGFP-N3, respectively, the RNA was extracted from the Sertoli cells and fibroblast cells. RT-PCR and PCR showed that a 294-bp fragment was present in cells with transfected plasmid pEGFP-N3HNP-1 but not in the control group cells (Figure 4). The PCR result showed that the HNP-1 gene has been integrated into the donor cell's genome.

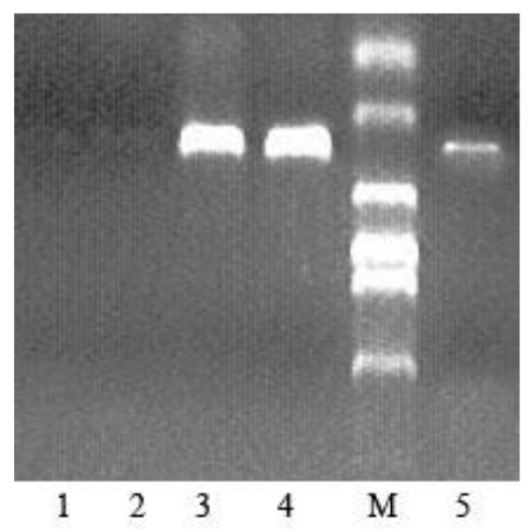

Figure 4. PCR analysis of positive clones. Lane $1=$ Sertoli cells transfected with vector pEGFP-N3; lane $2=$ fibroblast cells transfected with vector pEGFP-N3; lane $3=$ Sertoli cells transfected with vector pEGFP-N3-HNP-1; lane 4 = fibroblast cells transfected with vector pEGFP-N3-HNP-1; lane $5=\mathrm{HNP}-1 \mathrm{DNA}$; lane M= DL2000 marker.

\section{Cell fluorescence expression after transfection and transfection efficiency}

Fluorescence images are shown in Figure 5 at 24, 48, and $72 \mathrm{~h}$ after the two cell types were transfected with the pEGFP-N3-HNP-1 and pEGFP-N3 plasmids. At $24 \mathrm{~h}$, green fluorescence began to appear in fibroblast and Sertoli cells. The fluorescence intensity in control group cells transfected with pEGFP-N3 was significantly higher than that in the experimental group 
transfected with the PEGFP-N3-HNP-1 plasmid. The fluorescence intensity was strongest at $48 \mathrm{~h}$.

The transfection efficiency is shown in Table 2 at 24, 48, and $72 \mathrm{~h}$. The transfection efficiencies of fibroblast cells that had been transfected with the pEGFP-N3-HNP-1 and pEGFP-N3 plasmids were both higher than those of Sertoli cells $(\mathrm{P}<0.05)$. The transfection efficiency of fibroblast cells that had been transfected with the pEGFP-N3-HNP-1 plasmid was clearly lower than those transfected with the pEGFP-N3 plasmid $(\mathrm{P}<0.05)$. The transfection efficiency of Sertoli cells that had been transfected with the pEGFP-N3-HNP-1 plasmid was lower than that of cells transfected with the pEGFP-N3 plasmid $(\mathrm{P}<0.05)$.

The survival time of transfected cells is shown in Table 3. After transfection, the survival of fibroblast cells containing the pEGFP-N3-HNP-1 plasmid was significantly different $(\mathrm{P}<0.05)$ from cells containing the pEGFP-N3 plasmid, while the survival did not significantly differ $(\mathrm{P}<0.05)$ from that of Sertoli cells.

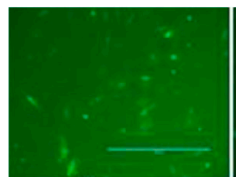

F1

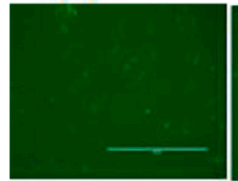

S1

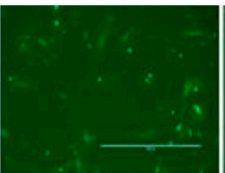

F2

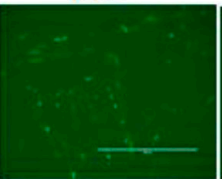

S2

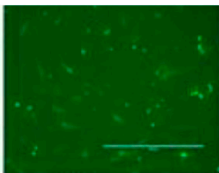

F3

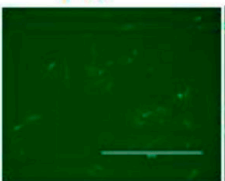

S3

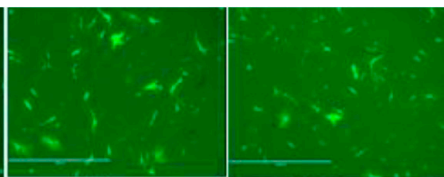

F4

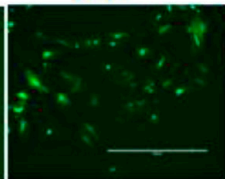

S4
F5

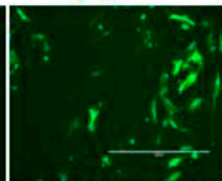

S5

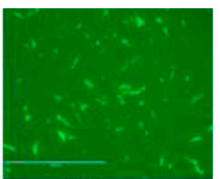

F6

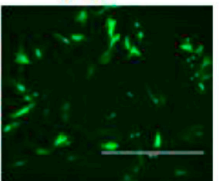

S6

Figure 5. Observation of the cell fluorescence expresstion at different times $(100 \mathrm{X}) . \mathrm{F}=$ fibroblast cells; $\mathrm{S}=\mathrm{Sertoli}$ cells. $1=$ transfected plasmid pEGFP-N3-HNP-1 at $24 \mathrm{~h} ; 2=$ transfected plasmid pEGFP-N3-HNP-1 at $48 \mathrm{~h} ; 3=$ transfected plasmid pEGFP-N3-HNP-1 at $72 \mathrm{~h} ; 4=$ transfected plasmid pEGFP-N3 at $24 \mathrm{~h} ; 5=$ transfected plasmid pEGFP-N3 at $48 \mathrm{~h} ; 6=$ transfected plasmid pEGFP-N3 at $72 \mathrm{~h}$.

Table 2. Cell transfection effciency at different times.

\begin{tabular}{|c|c|c|c|}
\hline \multirow[t]{2}{*}{ Name } & $24 \mathrm{~h}$ & $48 \mathrm{~h}$ & $72 \mathrm{~h}$ \\
\hline & Transfection efficiency (\%) & Transfection efficiency $(\%)$ & Transfection efficiency (\%) \\
\hline F-H & $6.89 \pm 0.14^{\mathrm{c}}$ & $10.65 \pm 0.24^{\mathrm{b}}$ & $9.08 \pm 0.17^{\mathrm{b}}$ \\
\hline F-N & $12.06 \pm 0.28^{\mathrm{a}}$ & $29.69 \pm 1.32^{\mathrm{a}}$ & $10.34 \pm 0.17^{\mathrm{a}}$ \\
\hline $\mathrm{S}-\mathrm{H}$ & $5.50 \pm 0.12^{\mathrm{d}}$ & $7.93 \pm 0.14^{\mathrm{c}}$ & $7.69 \pm 0.12^{\mathrm{c}}$ \\
\hline S-N & $7.84 \pm 0.11^{\mathrm{b}}$ & $9.62 \pm 0.12^{\mathrm{bc}}$ & $8.73 \pm 0.09^{b}$ \\
\hline
\end{tabular}

$\mathrm{F}=$ fibroblast cells; $\mathrm{S}=$ Sertoli cells; $\mathrm{H}=$ transfected with plasmid pEGFP-N3-HNP-1; $\mathrm{N}=$ transfected with plasmid pEGFP-N3. Groups with different letters were significantly different $(\mathrm{P}<0.05)$.

Table 3. Transfection effect at cell survival time.

\begin{tabular}{lccccc}
\hline & \multicolumn{2}{c}{ Fibroblast cells } & & \multicolumn{2}{c}{ Sertoli cells } \\
\cline { 2 - 3 } \cline { 5 - 6 } & pEGFP-N3-HNP-1 & pEGFP-N3 & & pEGFP-N3-HNP-1 & pEGFP-N3 \\
\hline Cell survival time (day) & $26 \pm 4.07^{\mathrm{b}}$ & $47.5 \pm 4.62^{\mathrm{a}}$ & & $19.5 \pm 2.79^{\mathrm{b}}$ & $23.5 \pm 3.94^{\mathrm{b}}$ \\
\hline
\end{tabular}

Groups with different letters were significantly different $(\mathrm{P}<0.05)$. 


\section{Behavior of transfected cells}

Cellular morphology and growth status changes were not obvious within $72 \mathrm{~h}$ after transfection. The appropriate concentration of G418 was added to the culture dishes at $72 \mathrm{~h}$ to screen for positive cells. The cells showed long spindles beginning 1 week later (Figure 6 F1, S1) and deformed to a polygon shape 2 weeks later (Figure 6 F2, S2). The early enhanced green fluorescent protein (EGFP) gene evenly filled the cytoplasm and nucleus (Figure 6 F1, F2, S1, S2) in 2 weeks, after which the cells stopped dividing. Three weeks later, the cell volume began to increase, irregular diffuse growth appeared, and long spindle and polygon shapes were observed. The cells elongated with pseudopodia, the intra cytoplasmic vacuoles increased (Figure 6 F3-F6, S3-S6), and the cells became more adherent so that they were no longer easily removed from the Petri dishes. The nucleus became round, the cytoplasm condensed, the chromatin edge of the nucleus began to shrink, and chromatin density increased. The cells became multinucleated, the cellular skeleton was deformed, EGFP localized in the nucleus, the fluorescence intensity in the nucleus was significantly higher than in the cytoplasm (Figure $6 \mathrm{~F} 4, \mathrm{~S} 3$ ), and the cells began losing the ability to divide; the cells died gradually. Negative cells showed no significant changes after 4 weeks of culture. The dying cells at 10 weeks are shown in Figure 6 F7-8, S7-8.
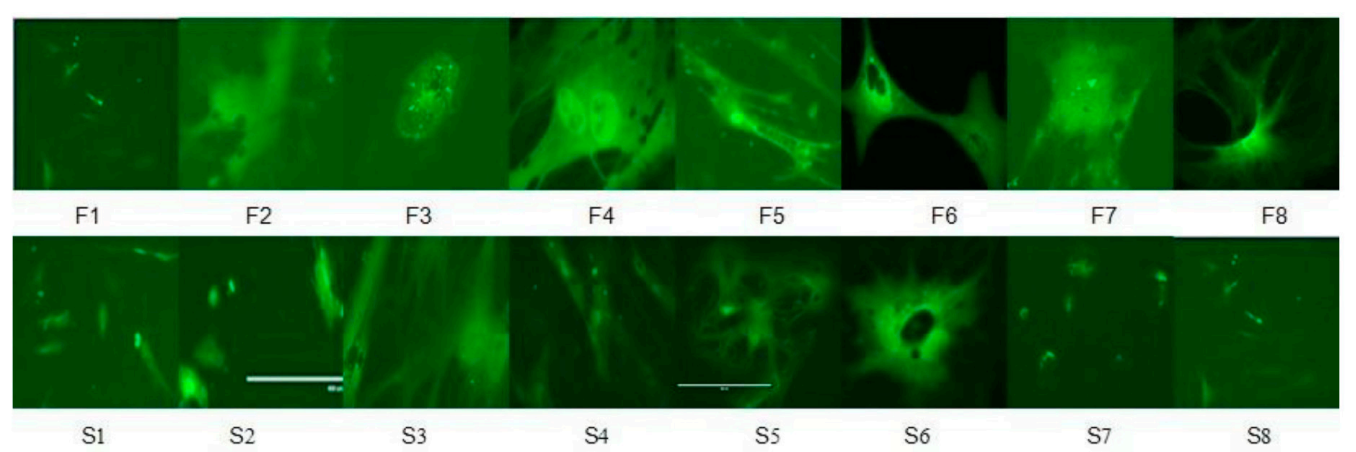

Figure 6. Observation of the cell $(10 \mathrm{X}$, stabe $400 \mu \mathrm{m}) . \mathrm{F}=$ fibroblast cells; $\mathrm{S}=$ Sertoli cells. $1=$ transfecion after 1 week; $2=$ transfecion after 2 weeks; $3-6=$ transfecion after 4 weeks; 7-8 $=$ transfecion at 10 weeks.

\section{DISCUSSION}

At $48 \mathrm{~h}$, EGFP expression was higher because the cells were in the relatively strong growth period and showed active protein synthesis, including synthesis of EGFP. Positive cells grew and divided after transfection. Most cells stopped dividing 2 weeks later. Nearly all cells lost the ability to divide 3 weeks later, and some cells lived for more than 2 months. The defense ability was clearly interfered with heterologous protein during normal cellular growth and division.

In Table 2, the transfection efficiencies of fibroblast cells containing the pEGFP-N3HNP-1 and pEGFP-N3 plasmids were both higher than the transfection efficiencies of Sertoli cells. The transfection efficiencies of cells transfected with the pEGFP-N3-HNP-1 plasmid for both cell types were clearly lower than those in cells transfected with the pEGFP-N3 plasmid. 
Thus, the ability of Sertoli cells to resist transfection was higher than that of fibroblast cells. This result may be closely related to the specific physiological function of Sertoli cells (Sun, 2011). The mammalian seminiferous tubule cavity is mainly composed of testicular Sertoli cells. The blood-testis barrier is composed of Sertoli cells, blood vessel endothelial basement membrane, connective tissue, and seminiferous tubule baseline, which are very closely associated (Syed and Hecht, 2002). The blood-testis barrier prevents the immune response (Arambepola et al., 1998). The ability of the two cell types to resist transfection of the pEGFP-N3-HNP-1 plasmid was higher than that for cells containing the pEGFP-N3 plasmid, which might be because of the different molecular weights of the pEGFP-N3-HNP-1 and pEGFP-N3 plasmids.

Table 3 shows that the survival of transfected fibroblast cells significantly differed $(\mathrm{P}<0.05)$ after transfection with the pEGFP-N3-HNP-1 and pEGFP-N3 plasmids, but the survival of transfected Sertoli cells was not significantly different $(\mathrm{P}<0.05)$. The injury to the Sertoli cells following transfection with $H N P-1$ had less of an impact than it did on fibroblast cells. Previous studies have shown that Sertoli cells contain various types of lysosomes that digest material in cells through active pinocytosis. Defensins not only have biological activity, but also show strong cytotoxicity. The cytotoxic activity of defensin depends in part on the target cell membrane, lipid composition, and the metabolic activity of target cells (Zhang and $\mathrm{Xu}, 2007)$. In addition, a variety of cytokines from Sertoli cells form a cytokine network in the testicles and adjust the immune function of the testis. Numerous studies have confirmed that the immunity function of the testes is sustained by the Sertoli cells (Whitmore et al., 1985; Cameron et al., 1990).

The survival of transfected fibroblast cells was significantly different $(\mathrm{P}<0.05)$ after transfection with the pEGFP-N3-HNP-1 and pEGFP-N3 plasmids. The cellular immune response system may have been induced when the exogenous genes entered the cell so that the injury to the Sertoli cells caused by transfection with HNP-1 was less than that to fibroblast cells.

In summary, the plasmid pEGFP-N3-HNP-1 was successfully constructed. The cells achieved maximum transfection efficiency at $48 \mathrm{~h}$. Two weeks after transfection, the cells began to lose the ability to divide. The ability of Sertoli cells to resist transfection was higher than that of fibroblast cells. The ability of the 2 cell types to resist transfection with the plasmid pEGFP-N3-HNP-1 was higher than that for the plasmid pEGFP-N3. The injury to Sertoli cells by transfection with the $H N P-1$ gene was reduced compared to fibroblast cells, which may be closely related with the specific physiological function of Sertoli cells.

\section{ACKNOWLEDGMENTS}

Research supported by the China National International Scientific and Technological Cooperation Project (\#2011DFA30760-2-1) and the Fund of Key Laboratory NEAU China (\#GXZDSYS-2012-07).

\section{REFERENCES}

Arambepola NK, Bunick D and Cooke PS (1998). Thyroid hormone and follicle-stimulating hormone regulate Müllerianinhibiting substance messenger ribonucleic acid expression in cultured neonatal rat Sertoli cells. Endocrinology 139: 4489-4495.

Cameron DF, Whittington K, Schultz RE and Selawry HP (1990). Successful islet/abdominal testis transplantation does not require Leydig cells. Transplantation 50: 649-653. 
Lehrer RI, Ferrari LG, Patterson-Delafield J and Sorrell T (1980). Fungicidal activity of rabbit alveolar and peritoneal macrophages against Candida albicans. Infect. Immunity 28: 1001-1008.

Lu W and de Leeuw E (2013). Pro-inflammatory and pro-apoptotic properties of human defensin 5. Biochem. Biophys. Res. Commun. 436: 557-562.

Sun X (2011). Research about testis Sertoli cells. Contemp. Anim. Husb. 11: 36-38.

Syed V and HechtNB (2002).Disruption of germ cell-Sertoli cell interactions leads to spermatogenic defects. Mol. Cell Endocrinol. 186: 155-157.

Whitmore WF 3rd, Karsh L and Gittes RF (1985). The role of germinal epithelium and spermatogenesis in the privileged survival of intratesticular grafts. J. Urol. 134: 782-786.

Wilde CG, Griffith JE, Marra MN, Snable JL, et al. (1989). Purification and characterization of human neutrophil peptide 4, a new member of the defensin family. J. Biol. Chem. 264: 11200-11203.

Yan Z and ChenX (2001). Research about paneth cell defensin. Foreign Medical Sciences 6: 189-193.

Yu L, Zheng P, Rong EG, Yu N, et al. (2013). Culture and identification of testis Sertoli cells in calf in vitro. J. Jilin Agricult. Univ. 35: 63-66.

Yu XH and Hu YF (2010). Biological characteristics and research progress of sertolicells. J. Clin. Rehabilitative Tissue Eng. Res. 14: 8311-8314.

Zhang Z andXu R (2007). Research and application of human denfensin. J. Radioimmunol. 20: 347-350.

Zheng P, Yu L, Tian YG, Huang He, et al. (2012). Isolation, purification and culture of male germline stem cells from neonatal bovine testis. J. Northeast Agric. Univ. 43: 32-38. 